\title{
A Case of Necrotizing Fasitis in a Patient Injecting Pomegranate Juice into Her Thigh
}

\author{
Cemil Ertürk*, Sabri Kerem Diril and Ahmet Şahin \\ Department of Orthopaedic Surgery, Faculty of Medicine, University of Health Sciences, Kanuni Sultan Suleyman Training and Research Hospital, \\ Kucukcekmece, Istanbul, Turkey
}

${ }^{*}$ Corresponding author: Cemil Ertürk, Assoc. Prof, Department of Orthopaedic Surgery, Faculty of Medicine, University of Health Sciences, Kanuni Sultan Suleyman Training and Research Hospital, Kucukcekmece, 34303 - Istanbul, Turkey; Phone: + 9021240415 00; Fax: + 902120212 5714790; Email: erturkc@yahoo.com

Received: August 30, 2019; Accepted: September 22, 2019; Published: September 26, 2019;

\section{Introduction}

Necrotizing Fasciitis (NF) is a disease characterized by rapidly spreading necrosis of soft tissues and fascia, which can lead to rapid death if not treated appropriately $[1,2]$. Etiology includes surgical incision, insect sting, incision, abrasion, contusion, injection, skin ulcer, perirectal abscess, incarcerated hernia, burn, splinter ingestion, birth and penetrating trauma [3]. In $70 \%$ of NF cases, the agent can be isolated in wound culture; $20 \%$ for blood culture. Grampositive bacteria are in the foreground and $70-90 \%$ of the cases are polymicrobial [4].

Early diagnosis, broad-spectrum antibiotic therapy and surgical debridement are essential. Despite treatment, 30\% of the patients die [5]. In this case report, we wanted to report the treatment of a NF patient who injected pomegranate juice with the thought of storing energy in his right thigh and right elbow of a drug addict, homeless and foreign national (Ukrainian Citizen) with serial debridement and skin grafting.

\section{Case}

A 36-year-old male patient of unknown nationality who was abandoned to the emergency department was consulted with the preliminary diagnosis of abscess in the extremities. In the examination of the patient; swelling, redness and ballotmaning of the right hip compared to the left hip. He also had an infected discharge wound on the anterior face of the right elbow. The patient's general condition was fond, orientated, cooperative. Glaskow coma score was 14 and neurological examination was normal. In laboratory tests CRP: $326 \mathrm{mg} / \mathrm{l}$, WBC: $23.1910^{3} / \mathrm{mcl}, \mathrm{Hb}: 12.9 \mathrm{~g} / \mathrm{dl}$, Na: 134mmol / L, creatinine: $0.8 \mathrm{mg} / \mathrm{dl}(70.7 \mathrm{mmol} / \mathrm{L})$, glucose: $122 \mathrm{mg} / \mathrm{dl}$ ( 11.3 $\mathrm{mmol} / \mathrm{L}$ ). In the presented case, the LRINEC (Laboratory Risk Indicator for Necrotizing Fasciitis) score was calculated to be 8 during the first application (Table I).

On radiological examinations, X-ray radiography showed gas shadows compatible with NF in the right thigh region (Figure 1). MRI; Fluid collections were observed starting from the right gluteal region and extending to the proximal of the thigh, the largest of which was approximately $6.5 \times 4 \mathrm{~cm}$ lateral to the proximal section of the thigh (abscess?) (Figure 2). Superficial tissue USG; There were diffuse edema findings in the right arm and a $45 \mathrm{~mm}$ collection-abscess.
Table 1. Laboratory Risk Indicators for Necrotizing Fasciitis (LRINEC).

\begin{tabular}{|c|c|c|}
\hline Value & LRINEC score & Values of the case \\
\hline \multicolumn{3}{|l|}{ C-reaktif protein (mg/L) } \\
\hline$<150$ & 0 & 326 \\
\hline$>150$ & 4 & \\
\hline \multicolumn{3}{|l|}{ White blood cell count (cell $/ \mathrm{mm}^{3}$ ) } \\
\hline$<150$ & 0 & 23 \\
\hline $15-25$ & 1 & \\
\hline$>25$ & 2 & \\
\hline \multicolumn{3}{|l|}{ Hemoglobin level (g/dL) } \\
\hline$>13.50$ & 0 & 12.9 \\
\hline $11-135$ & 1 & \\
\hline$<11$ & 2 & \\
\hline \multicolumn{3}{|l|}{ Sodium level (mmol/L) } \\
\hline$\geq 135$ & 0 & 134 \\
\hline$<135$ & 2 & \\
\hline \multicolumn{3}{|l|}{ Creatinine level (mg/dL) } \\
\hline$\leq 1.6$ & 0 & 0.8 \\
\hline$>1.6$ & 2 & \\
\hline \multicolumn{3}{|l|}{ Olucoie dOzeyi (mg/dL) } \\
\hline$\leq 180$ & 0 & 122 \\
\hline$>180$ & 1 & \\
\hline
\end{tabular}

Emergency debridement was planned in the emergency department within 6-8 hours due to worsening of the general condition, high infection parameters, hemodynamic instability, change in consciousness and septic shock. Radical debridement, abscess drainage, abundant washing was performed and culture samples were taken in the operating room conditions (Figure 3,4). He was transferred to the postoperative intensive care unit. Subsequently, serial debridement was performed 5 times at 48 hour intervals. At each debridement stage, the patient was evaluated for amputation. In this process, he was treated by infectious diseases according to the patient's 
clinic, blood parameters and culture results. Two weeks later, when he did not need intensive care, he was transferred to the orthopedic clinic. The patient underwent re-debridement and a Vacuum-Assisted Wound Care System (VAC) was applied.

In the wound culture sample, Escherichia Coli was produced. Methicillin-resistant Staphylococcus epidermidis was isolated in blood cultures. One week later, Acinetobacter Baumannii was isolate in blood culture too. 2 weeks after admission CRP was $141 \mathrm{mg} / \mathrm{l}$,
WBC was $11.99 * 10^{3} / \mathrm{mcl}, \mathrm{Hb}$ value was $8.3 \mathrm{~g} / \mathrm{dl}$, Na: $137 \mathrm{mmol} / \mathrm{L}$, $\mathrm{Kr}: 0.44 \mathrm{mg} / \mathrm{dl}$ (38.5 mmol / L), glucose: $77 \mathrm{mg} / \mathrm{dl}$ (7.13 mmol / L). LRINEC score was 2.

As a result of the complete disappearance of infection findings 1.5 months later, wound areas were closed with skin grafts taken from the opposite thigh (Figure 5,6). After the lack of medical needs of the patient for lack permission to remain in Turkey it was deported by mobile discharged. The whole process took 2.5 months.

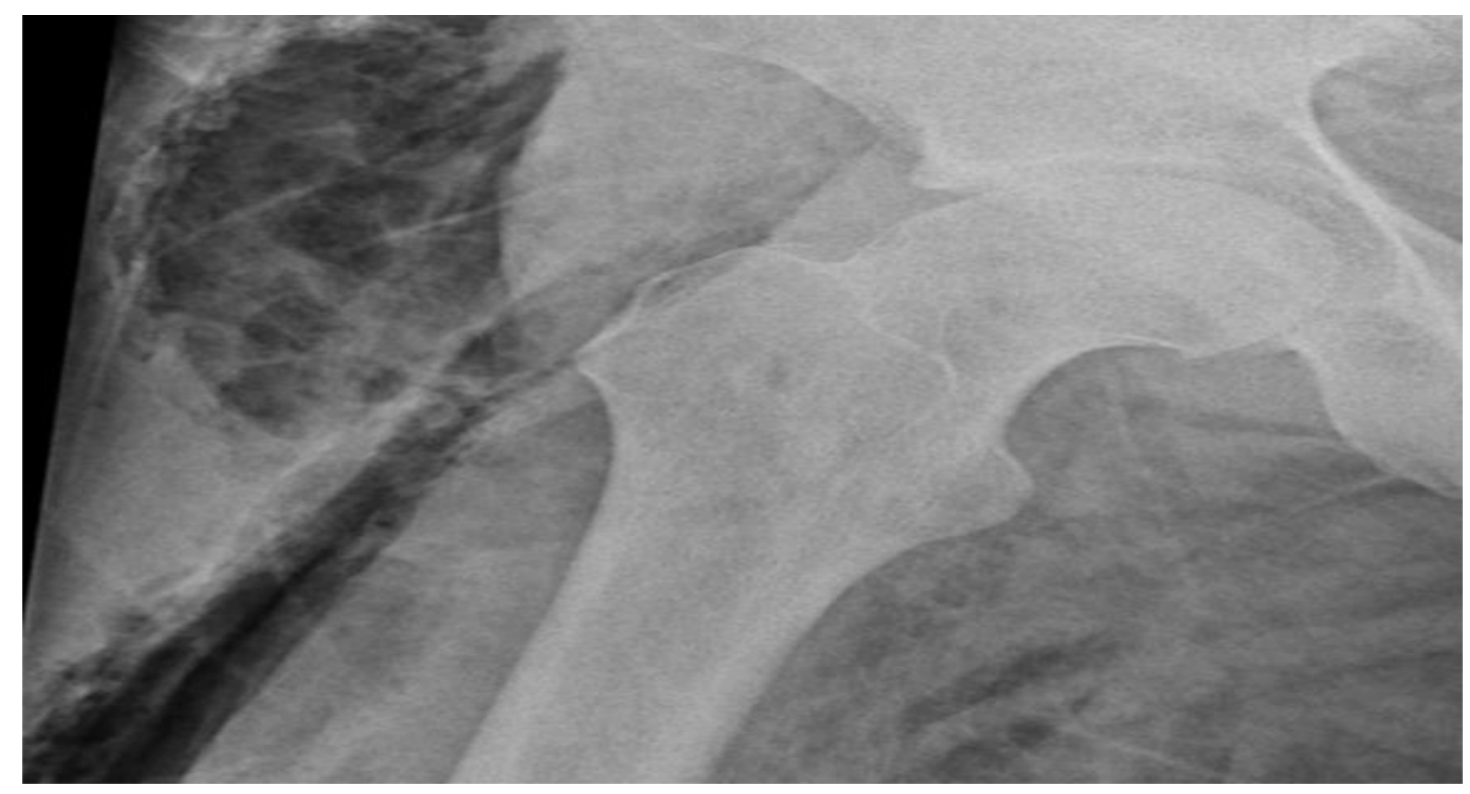

Figure 1. X-ray image of gas shadows on patient's right thigh

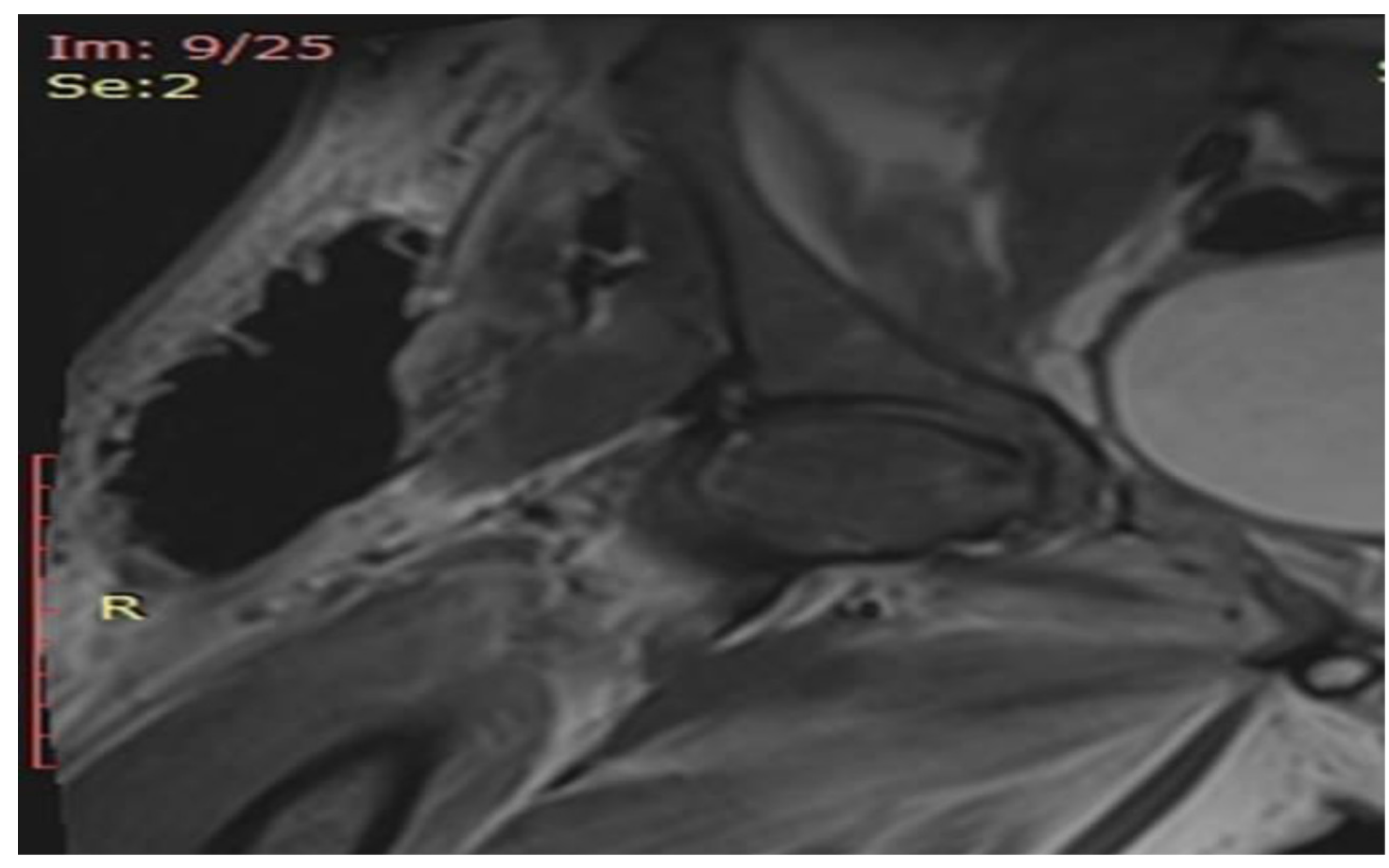

Figure 2. MRI image of abscess on the right thigh of the patient 
Ertürk C (2019) A Case of Necrotizing Fasitis in a Patient Injecting Pomegranate Juice into Her Thigh

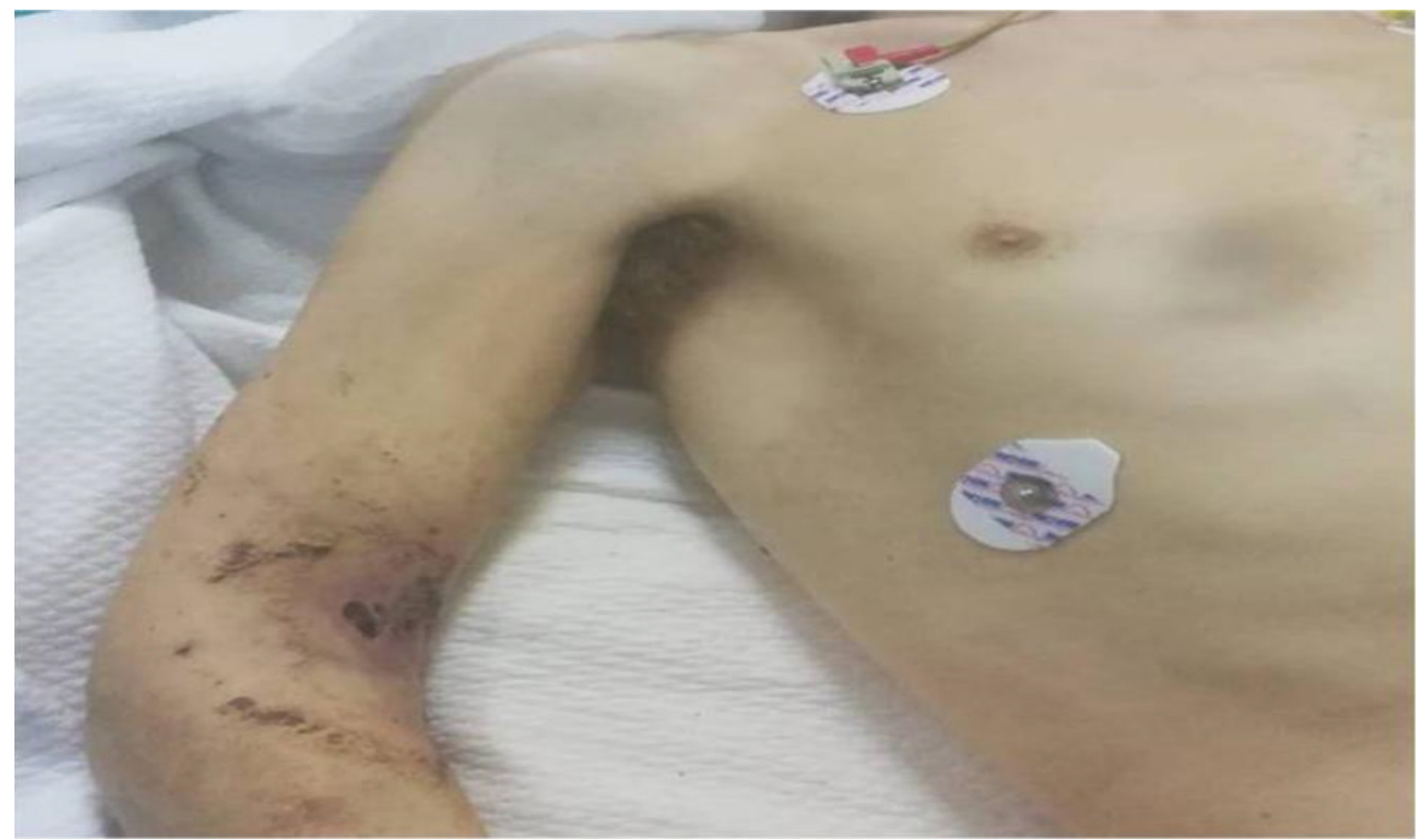

Figure 3: Drained abscess on the right elbow of the patient

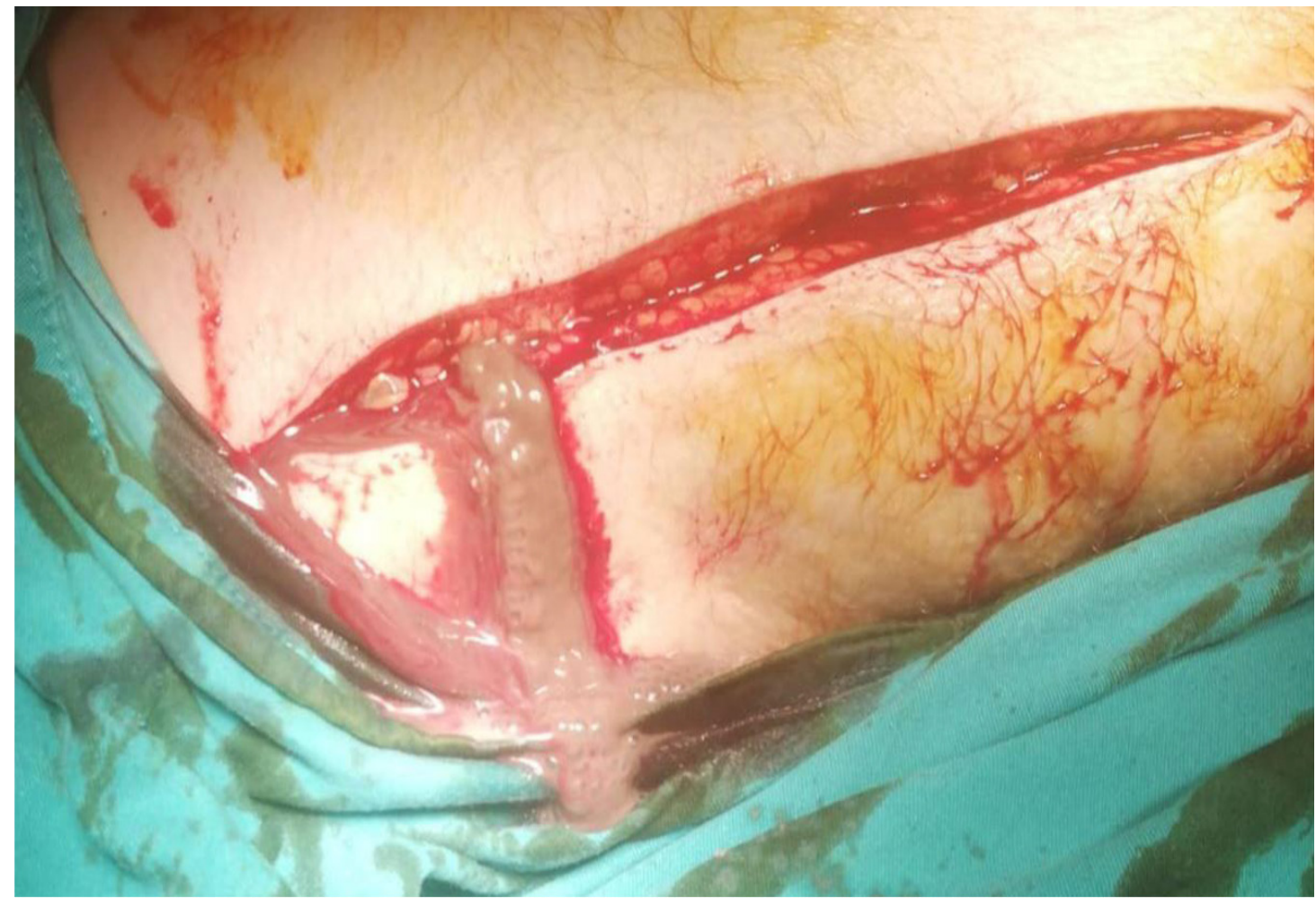

Figure 4. Perop right thigh drainage image 


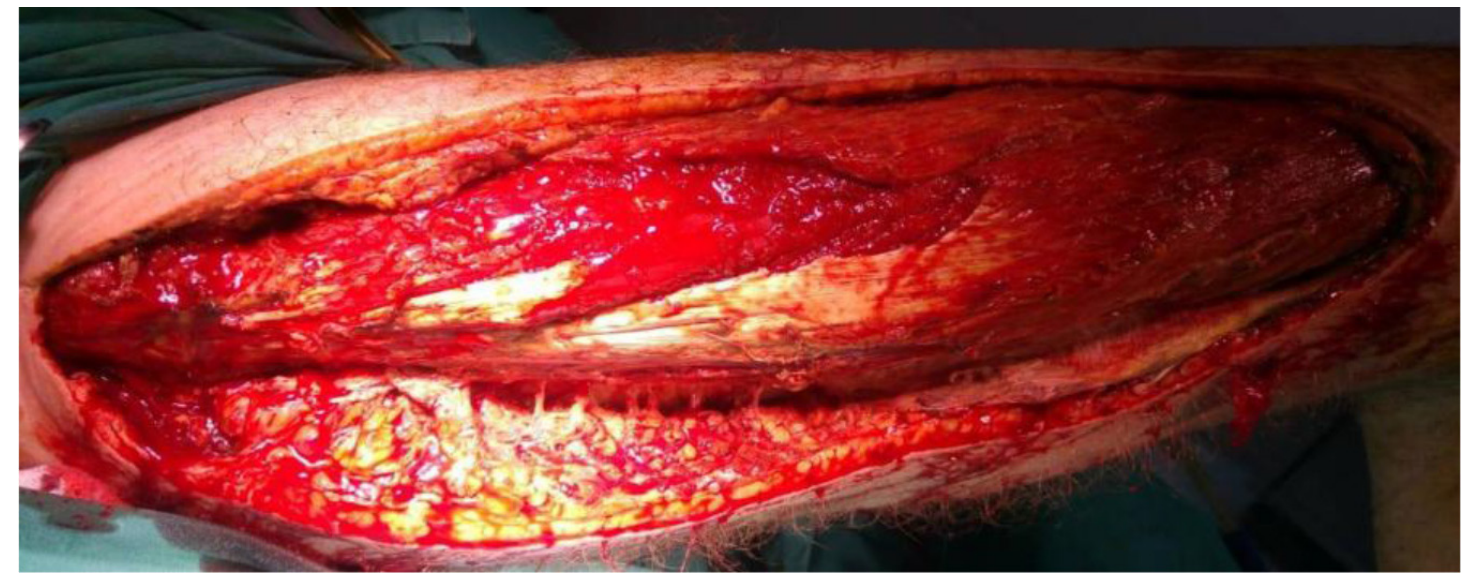

Figure 5. Right thigh image after serial debridements

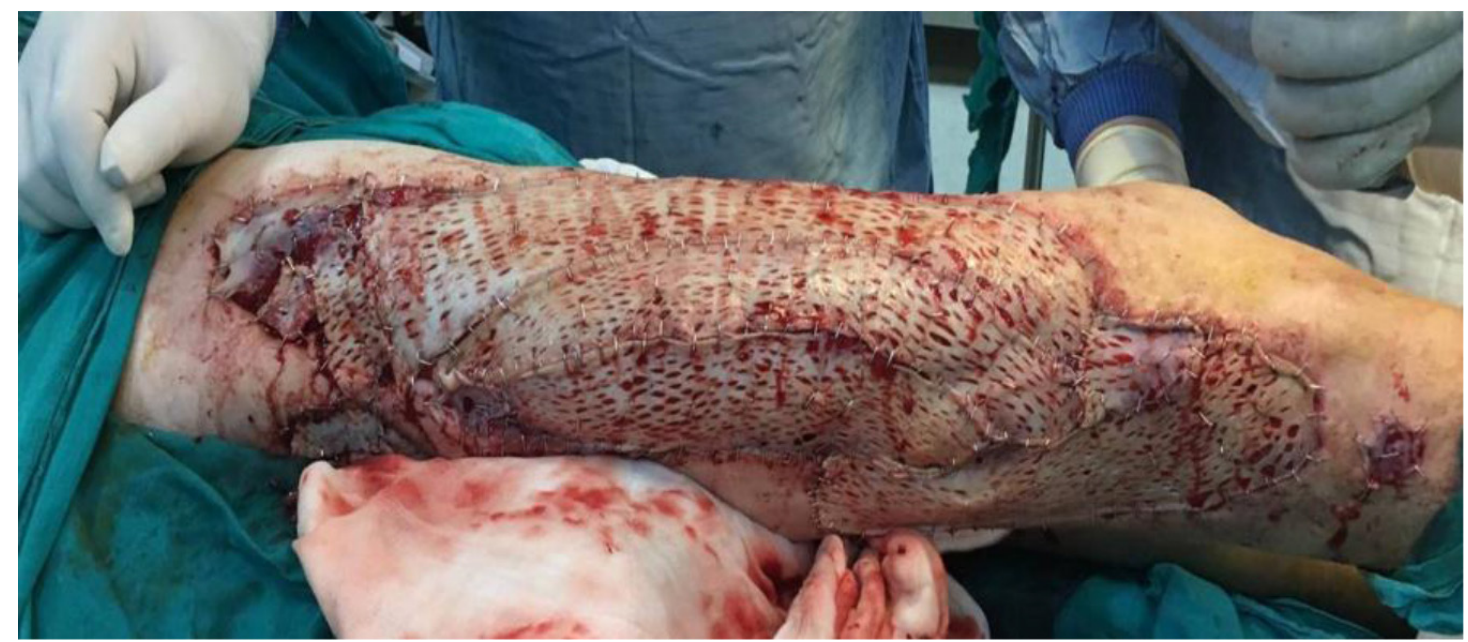

Figure 6. Appearance of the thigh after grafting

\section{Discussion}

Although rare, every surgeon treats at least one NF case throughout his life [6]. Rapidly spreading necrosis can cause systemic sepsis, toxic shock syndrome and multiorgan failure [7]. The patient was in septic shock when we operated.

LRINEC risk score was accepted as high risk with 8 points at the first admission [8]. Early, aggressive treatment is required and necrotizing fasciitis is an surgical emergency [9]. The patient should be evaluated as a whole and the decision of amputation should be reviewed at every stage. The recommended intravenous antibiotic therapy depends on the etiological factors; however, clindamycin, penicillin and thirdgeneration cephalosporins can be started empirically [10].

Delay of the first debridement may increase mortality up to $71 \%$ [11]. Although we considered the possibility of amputation after each debridement in our case, we have always emphasized limb sparing surgery [3]. In addition, the length of hospital stay in NF cases brings a significant financial burden [12].

As a result; Many problems were dealt with during the diagnosis, treatment and discharge phase of the patient, who was a foreign national, had no relatives and no insurance. We tried to apply the early diagnosis, antibiotic therapy and surgical debridement approaches, which are the principle of NF, appropriately. After serial debridement, wound and blood culture, antibiotic therapy and VAC treatment, skin grafting was applied to the wounds. With the treatment principles, the patient's survival and return to life were achieved.

\section{References}

1. Trent JT, Kirsner RS (2002) Diagnosing necrotizing fasciitis. Adv Skin Wound Care 15: 135-8.

2. File TM Jr, Tan JS, DiPersio JR (1998) Group A streptococcal necrotizing fasciitis. Diagnosing and treating the "flesh-eating bacteria syndrome". Cleve Clin J Med 65: 241-9.

3. Carter PS, Banwell PE (2004) Necrotising fasciitis: a new management algorithm based on clinical classification. Int Wound J 1: 189-98.

4. Shaikh N, El-Menyar A, Mudali IN, Tabeb A, Al-Thani H (2015) Clinical presentations and outcomes of necrotizing fasciitis in males and females over a 13-year period. Ann Med Surg (Lond) 4: 355-60.

5. Sun X, Xie T (2015) Management of Necrotizing Fasciitis and Its Surgical Aspects. Int J Low Extrem Wounds. 14: 328-34.

6. Naqvi GA, Malik SA, Jan W (2009) Necrotizing fasciitis of the lower extremity: a case report and current concept of diagnosis and management. Scand J Trauma Resusc Emerg Med Pg No: 17-28.

7. Fichev G, Kostov V, Marina M, Tzankova M (1997) Fornier's gangrene: a clinical and bacteriological study. Anaerobe 3: 195-7. 
Ertürk C (2019) A Case of Necrotizing Fasitis in a Patient Injecting Pomegranate Juice into Her Thigh

8. Wong CH, Khin LW, Heng KS, Tan KC, Low CO (2004) The LRINEC (Laboratory Risk Indicator for Necrotizing Fasciitis) score: a tool for distinguishing necrotizing fasciitis from other soft tissue infections. Crit Care Med 32: 1535-41.

9. McDonald LS, Shupe PG, Raiszadeh K, Singh A (2014) Misdiagnosed pneumothorax interpreted as NF of the chest wall: case report of potentially prevntable death. Patient Saf Surg 8: 20.

10. Shaikh N, El-Menyar A, Mudali IN, Tabeb A, Al-Thani H (2015) Clinical presentations and outcomes of necrotizing fasciitis in males and females over a 13-year period. Ann Med Surg (Lond) 4: 355-60.
11. Kuncir EJ, TillouA, Hill CR, Ptrone P, Kimbrell B, Asencio JA (2003) Necrotizing soft tissue infections. Emerg Med Clin North Am 21: 1075-87.

12. Widjaja AB, Tran A, Cleland H, Leung M, Millar I (2005) The hospital costs of treating necrotizing fasciitis. ANZ J Surg 75: 1059-64.

\section{Citation:}

Ertürk C, Diril SK, Sahin A (2019) A Case of Necrotizing Fasitis in a Patient Injecting Pomegranate Juice into Her Thigh. Integr J Orthop Traumatol Volume 2 (4): 1-5. 